\title{
News on Dark Matter from ICRC 2021
}

\author{
Marco Taoso ${ }^{a, *}$ \\ ${ }^{a}$ I.N.F.N. sezione di Torino, \\ via P. Giuria 1, I-10125 Torino, Italy \\ E-mail: marco.taoso@to.infn.it
}

This document is based on a rapporteur talk given at the $37^{\text {th }}$ International Cosmic Ray Conference (ICRC 2021). The article summarises the status of DM searches.

$37^{\text {th }}$ International Cosmic Ray Conference (ICRC 2021)

July 12th-23rd, 2021

Online - Berlin, Germany

\footnotetext{
*Presenter
} 


\section{Introduction}

Numerous and compelling evidences for dark matter (DM) emerge from cosmological and astrophysical probes. This wealth of observations provide many precious information on DM, like its abundance and distribution, and they further provide constraints on some DM properties. On the other hand, the nature of DM is still unknown, and many possibilities for DM candidates still exist. For instance the DM mass is very weakly constrained. DM can be composed by ultra-light scalar particles with masses down to $10^{-21} \mathrm{eV}$, or even by macroscopic objects. DM candidates can often lead to signals in astrophysical environments, which can be searched for with current and future experiments. The most famous example is the one of Weakly Interacting Massive Particles (WIMPs). WIMPs are DM candidates whose abundance in the early Universe is controlled by pair annihilations. If the final states of the annihilation processes are Standard Model (SM) particles, these processes offer a way to probe WIMPs. This indirect detection strategy aims at detecting photons, neutrinos and charged particles produced by DM annihilations inside the cosmological structures. Furthermore, the cosmological abundance of DM provides an indication for the value of the DM annihilation cross-section. In fact, under certain assumptions, WIMPs inherit the correct relic abundance if they annihilate in the early Universe with a thermal relic cross-section $\langle\sigma v\rangle \simeq 310^{-26} \mathrm{~cm}^{3} \mathrm{~s}^{-1}$. If $\langle\sigma v\rangle$ is velocity independent, the number mentioned above, can be considered as a valuable benchmark also for the processes occurring in astrophysical environments. Of course, this benchmark should be taken with a grain of salt, since exceptions to this scenario often occur in WIMP models, e.g. velocity dependent $\langle\sigma v\rangle$ or coannihilations. Other strategies to detect WIMPs consist on producing them at accelerators, or detecting the scattering process of DM with the material of a detector. The latter strategy is the so-called direct detection method.

In this article, I will provide a brief overview of the contributions on DM searches presented at ICRC 2021. Given the main focus of the conference, much attention has been devoted to indirect searches, especially of WIMPs. However, as discussed below, some contributions on direct detection and non-WIMP DM have also been presented. For the sake of conciseness, I will refer only to the contributions presented at ICRC 2021. However, further references and details can be found in those contributions.

The document is organised as follows: sec. 2 is devoted to indirect searches of DM with photons. Sec. 3 and sec. 4 focuses on DM searches with charged cosmic-rays and neutrinos respectively. A brief discussion on direct detection searches is in sec. 5 . Sec. 6 concludes.

\section{DM searches with photons}

\subsection{Dwarf spheroidal galaxies and subhalos}

Dwarf spheroidal galaxies are among the most promising targets for indirect dark matter searches. Their high mass-to-light ratios reveal that they are DM dominated objects. Moreover, their negligible astrophysical background, and their locations at high Galactic latitudes, make them clean environments at gamma-ray energies. Searches for DM signals in these galaxies have been performed by the space-borne Fermi-Large Area Telescope (Fermi-LAT), the imaging atmospheric Cherenkov telescopes (IACT) arrays H.E.S.S., MAGIC, and VERITAS, and the water Cherenkov array HAWC. These observations are complementary in the energy range explored, and targets 

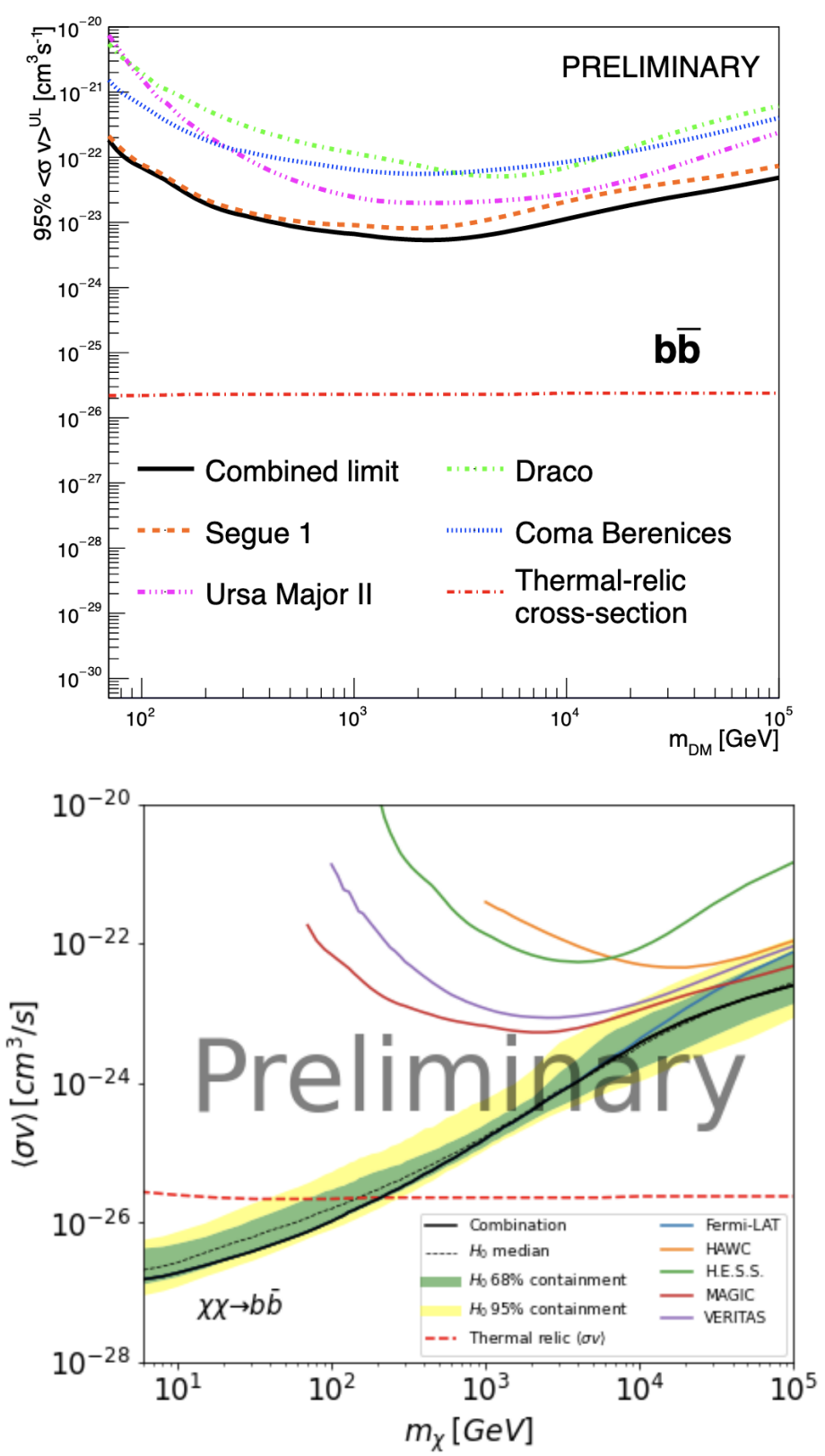

Figure 1: Top panel: $95 \%$ confidence level upper bounds on the DM annihilation cross-section from observations of dwarf spheroidal galaxies performed by MAGIC. Taken from [2]. Bottom: Upper limits at $95 \%$ confidence level on the DM annihilation cross-section $\langle\sigma v\rangle$ as a function of the DM mass from a combined analysis of Fermi-LAT, H.E.S.S., MAGIC, VERITAS and HAWC observations of dwarf spheroidal galaxies. Taken from [1].

observed. Fermi-LAT has a wide field of view, scanning the whole sky every three hours, and detects photons in the energy $\sim 20 \mathrm{MeV}-1 \mathrm{TeV}$. IACTs cover higher energies (from $\sim 30-85 \mathrm{GeV}$ up to tens or several hundreds of $\mathrm{TeV}$, depending on the experiment) and have smaller fields of view. Moreover, in general, the three experiments mentioned above observe different targets due to 
their different locations. HAWC covers energies from $\sim$ from $300 \mathrm{GeV}$ up to $\sim 100 \mathrm{TeV}$, with the advantage over the IACTs of a the larger instant sky coverage, and high duty cycle. A combined analysis of the individual searches performed by these experiments has been presented in [1]. The search is based on observations of twenty classical and ultrafaint dwarf spheroidal galaxies, most of them observed by multiple experiments. This joint analysis allows to increase the statistics, and thus the sensitivity to DM signals, further allowing a common treatment of several systematic uncertainties, for instance the one associated with the modelling of the DM density distribution in the targets. No significant excesses have been found in the data, and upper limits on the DM annihilation cross-section have been obtained for several annihilation channels, see fig. 1 for the $b \bar{b}$ channel. As shown in the figure, the constraints are largely driven by the Fermi-LAT for DM masses below $\sim 10 \mathrm{TeV}\left(\sim 500 \mathrm{GeV}\right.$ for the $\tau^{+} \tau^{-}$channel) while IACTs and HAWC take over at larger masses. The combined upper limits are 2-3 times more stringent than the bounds derived from individual experiments.

New results obtained from searches of DM with MAGIC have been presented in [2]. The analysis is based on recent observations of the Draco and Coma Berenices dwarf spheroidal galaxies, combined with the ones of the previously observed Segue 1 and Ursa Major II galaxies. No DM signals have been found, and upper limits on the DM annihilation cross-section have been derived, see fig. 1. The bounds are dominated by the Segue 1 contribution. Still the combined analysis allows to improve the upper limits, and mitigates the effects from systematic uncertainties related to the target.

Ref. [3] reported an analysis based on observations of dwarf-spheroidal galaxies taken from 2007 to 2013 by VERITAS. The dataset is reanalized taking into account the angular extension of the sources. This additional information is expected to improve the constraints.

Other potential targets for DM searches are dwarf irregular galaxies, which, as dwarf-spheroidal galaxies, are DM dominated systems. A search for DM signals with Fermi-LAT has been presented in [10]. The upper limits on the DM annihilation cross-section are less stringent than those from dwarf-spheroidal galaxies.

Finally, DM signals can be produced by dark subhalos. In fact, the hierarchical formation scenario of the cosmological structures, predicts that a population of dark subhalos should be present in DM halos like that one that hosts our galaxy. At ICRC 2021, the properties and relevance of these substructures for DM searches have been discussed in [4-9]. Ref.[5] presented a search for DM subhalo candidates among the Fermi-LAT unidentified sources. After applying selection criteria and implementing a spectral analysis to test the DM origin of the sources, no preference for DM has been found. Furthermore, exploiting N-body simulations, ref.[5] examined the expected spatial extension of the sources, and their impact on the Fermi-LAT sensitivity to DM signals. The upper limits on the DM annihilation cross-section obtained from this analysis are complementary (although weaker) to the ones from dwarf spheroidal galaxies discussed above.

\subsection{Galactic center}

Since 2019 an excess of gamma-rays has been reported in the Fermi-LAT data from the galactic center region. The emission is spatially extended and peaks at energies around few GeVs. The origin and properties of the excess have been investigated extensively in the literature, see [13-15] and references therein. The most discussed explanations are in terms of DM annihilations, or 



Figure 2: Top: Upper limits on the DM annihilation cross section into $W^{+} W^{-}$from a DM search at the galactic center region with H.E.S.S. Taken from [11]. Bottom: Upper limits on the DM annihilation cross section into $\gamma \gamma$ from a search of spectral lines performed by MAGIC. Taken from [12].

an unresolved population of millisecond pulsars (MSPs) in the galactic bulge of our galaxy. At ICRC-2021 ref. [13] presented an updated analysis on the DM interpretation, in the light of current multi-wavelength constraints. According to this study, an explanation in terms of DM candidates annihilating into hadronic channels is in tension with constraints from anti-protons, while viable solutions emerge for other scenarios, e.g. annihilations into $\mu^{+} \mu^{-}$. 
Currently, it is still under debate whether data favour one of two hypothesis mentioned above, i.e. DM annihilations or astrophysical sources. Interestingly, future multi-wavelength surveys of the galactic center region could be able to test the MSP interpretation, leading to the detection of currently unresolved sources. In particular, ref. [14] finds that hundreds of sources could be detectable in X-rays by the Chandra X-ray Observatory. Furthermore, analyzing Chandra data, ref. [14] identified promising MSPs candidates among the currently unidentified sources, and further characterized their expected emission at radio wavelengths, motivating future radio observations which could detect the pulsation of the sources and confirm their MSP nature.

Another route to test the MSP hypothesis is to exploit the high energy electrons and positrons that these sources are expected to inject in the interstellar medium. These particles produce gamma-rays through Inverse Compton processes, leading to an high-energy tail in the spectrum of the galactic center emission. Ref. [15] has explored the prospects for detection of such a signal with the forthcoming Cherenkov Telescope Array (CTA). According to this analysis, a detection could be possible, depending on the properties of the MSPs, in particular their efficiency in injecting electron and positrons. The morphological property of the emission could be a key property to distinguish the MSP interpretation of the excess from the DM one.

Searches of DM signals from the galactic center region have been presented by the MAGIC [12] and H.E.S.S. [11] collaborations. Ref. [11] presented an analysis based on 546 hours of observations performed between 2014 and 2020. The region of interest for the search extends up to 2.9 degrees from the galactic center. No significant excesses have been found, and upper limits on the DM annihilation cross-section have been obtained, see fig. 2 for DM annihilations into $W^{+} W^{-}$. These limits, derived assuming an Einasto DM density distribution, improve previous H.E.S.S. constraints, and are almost at the level of the thermal relic cross section for DM masses in the TeV range.

MAGIC has performed a search for spectral lines using about 200 hours of observations of the galactic center region [12]. Such monochromatic emissions are expected, for instance, in models where $\mathrm{DM}$ is in form of $\mathrm{TeV}$ scale electroweak multiplets, see e.g. [18]. Given their location, the MAGIC telescopes observe the galactic center at large zenith angles, which leads to an increase of the energy threshold, but also an improvement of the sensitivity at energies above several $\mathrm{TeV}$. Upper limits on the DM annihilation cross section into two photons are shown in fig. 2, assuming two different DM density distributions: an Einasto profile and a cored profile. These constraints are competitive with the ones obtained by other experiments. Given the relatively small region of interest considered in the analysis, it turns out that the limits are quite sensitive to the choice of the DM density profile, see fig. 2. For the same reason, the DM distribution plays a crucial role also in the limits obtained in [11].

Given its large field of view and continuous duty cycle, HAWC is a suitable instrument to perform large sky surveys. Ref. [19] reported a search of DM signals from the galactic halo, leading to constraints on the DM parameter space. The results have been presented both for annihilating DM, and for decaying DM. For the latter, the signal arises from the decay of long-lived DM candidates into SM particles. The limits are complementary to the ones from other experiments (especially for decaying DM), and their dependence on the DM density profile is mitigated by the large region of interest considered in the analysis. Other DM searches with HAWC have been presented in [20,21] focussing on the diffuse gamma-ray background, and clusters of galaxies. 

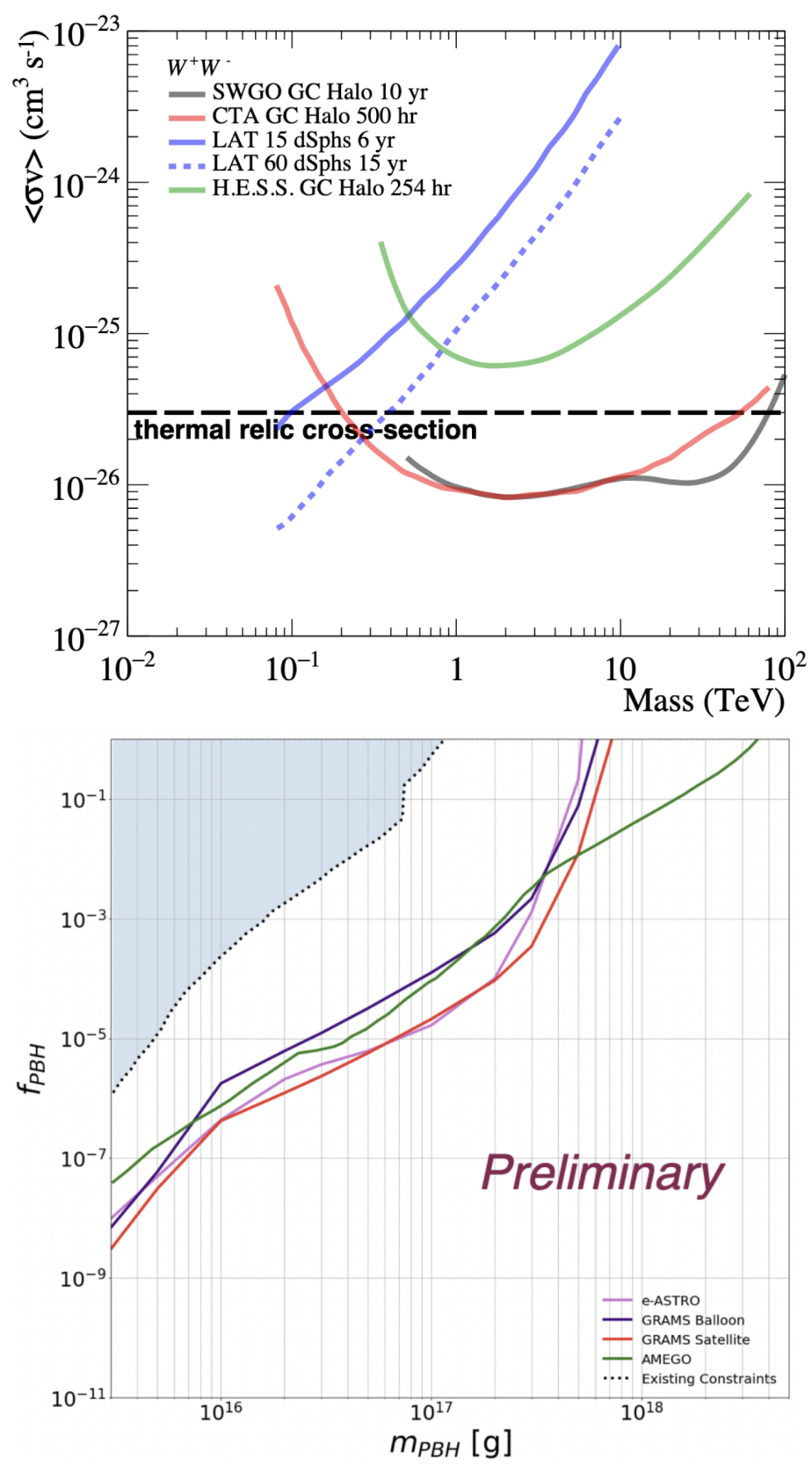

Figure 3: Top: Expected sensitivity of SWGO for DM annihilations. Taken from [16]. Bottom: Expected upper limits of GRAMS and other experiments on the fractional abundance of PBHs. Taken from [17].

The Large High Altitude Air Shower Observatory (LHAASO) offers unprecedented sensitivity to very high energy gamma-rays. It is an hybrid extensive air shower experiment for gamma-ray astronomy and cosmic-rays physics, operating since 2019, exploiting different types of detectors, and composed by three sub-arrays. LHAASO has a large field of view, covers $60 \%$ of the sky each day, and detects gamma-rays from several hundreds of $\mathrm{GeV}$ up to several $\mathrm{PeV}$ energies. At ICRC 2021, the relevance of LHAASO for DM searches has been explored in [22, 23]. Ref.[22] 
presented an analysis performed with the KM2A array, which covers gamma-ray energies above 10 $\mathrm{TeV}$. The search is based on about 340 days of data, and focuses on DM signals from the galactic halos. No significant excesses have been found. These results have been used to constrain heavy DM candidates, with masses above $10^{5} \mathrm{GeV}$, decaying into a variety of SM final states. The limits are competitive with those from other experiments, excluding new regions of the DM parameter space. The decay of heavy DM has been proposed in the literature to explain part of the high-energy astrophysical neutrinos detected by IceCube, see the discussion in [24]. Ref.[22] shows that (for the $\tau^{+} \tau^{-}$and $b \bar{b}$ channels) this explanation is in tension with the bounds from LHAASO and other probes.

\subsection{Prospects for future gamma-ray detectors}

The forthcoming CTA experiment will be one of the leading high-energy gamma-ray instruments. It is a ground based gamma-ray observatory composed by more than one hundred telescopes, located in two sites in the northern and southern hemispheres. It will cover a wide energy range, from about $20 \mathrm{GeV}$ up to $300 \mathrm{GeV}$, with an excellent angular resolution, down to arcminute scales. At ICRC 2021, prospects for DM searches with CTA have been discussed focussing on the galactic center region [25], the Perseus cluster of galaxies [26], and DM subhalos [4]. The analysis of [25] assumes a survey of the galactic center region with about 500 hours of observations, and it is based on a template fitting approach, where a series of signal and backgrounds templates are fitted to the (simulated in this case) data. This method differs from the ON/OFF technique, more conventional for IACTs, where two different regions are selected (with larger and smaller expected signal fluxes, respectively for the ON and OFF regions), and used to separate the signal from the background. The potential advantage of the template fitting method for this search has been discussed. Assuming an Einasto profile for the DM density distribution, ref. [25] shows that CTA will reach unprecedented sensitivity to DM signals, exploring thermal relic cross sections for a broad range of masses in the $\mathrm{TeV}$ domain. The expected limits weaken significantly for a DM distribution with a core in the inner kpc region of our galaxy. Ref. [25] investigates how this effect can be mitigated including additional data from an extended survey above the galactic plane (reaching latitudes up to 15 degrees).

The southern wide field-of-view gamma-ray observatory (SWGO) is a proposed extensive air shower experiment to be located in the southern hemisphere [16]. SWGO will cover energies from $100 \mathrm{GeV}$ up to $100 \mathrm{TeV}$, it will have a large field of view, and a significantly better sensitivity than HAWC, which is based on a similar concept for gamma-ray detection. Ref. [16] reported future sensitivities for SWGO to DM signals from the inner galactic halo and dwarf spheroidal galaxies. Fig. 3 shows the results for DM annihilations into $W^{+} W^{-}$. The sensitivity reach is compared to the one for CTA, current upper limits from Fermi-LAT and H.E.S.S., and projected sensitivities for Fermi-LAT with future DM searches from dwarf spheroidal galaxies. As evident, SWGO will be complementary to CTA, and the combination of these different experiments will allow to test thermal relic cross sections up to masses almost of $100 \mathrm{TeV}$.

Several gamma-ray experiments have been proposed in the recent years to explore the so-called $\mathrm{MeV}$ gap. This refers to the energy range between $\sim 100 \mathrm{keV}$ up to $20 \mathrm{MeV}$. The regimes at higher and lower energies have been extensively investigated respectively by Fermi-LAT, and X-ray instruments like NuSTAR. The intermediate region, the $\mathrm{MeV}$ gap, is instead far less explored, by experiments like COMPTEL which had however significantly smaller sensitivities than the other 
mentioned telescopes. The relevance of future $\mathrm{MeV}$ gamma-ray experiments for $\mathrm{DM}$ searches have been discussed in [17, 27], focussing respectively on the GRAMS and GECCO proposals. These experiments have the potential to significantly improve the reach of current probes to sub-GeV DM. Moreover, ref.[27] shows that GECCO could play an important role to shed light on the nature of the $511 \mathrm{keV}$ line from the galactic bulge. This emission arises from the annihilation of low-energy electrons and positrons. The origin of these positrons is still unknown, and dark matter has been proposed as a possible source. Given its good sensitivity and angular resolution, GECCO should be able to distinguish a truly diffuse emission, like the one from dark matter, from the one induced by a population of point-sources.

While we wait for future observations at $\mathrm{MeV}$ energies, different strategies can be envisaged to constrain light DM with current data. Ref.[28] proposes to exploit the Inverse Compton emission produced by electrons and positrons produced by DM annihilations. For sub-GeV DM, these fluxes peak in the high X-ray band, and they can be constrained by INTEGRAL observations. Ref.[28] finds that competitive bounds can be inferred for DM masses in the range $0.1 \mathrm{MeV}-1.5 \mathrm{GeV}$.

\subsection{Beyond the standard WIMPs}

DM could belong to a dark sector containing new states which interact feebly with the SM. Furthermore, the abundance of DM could be set by annihilation processes into light metastable particles of the dark sector. In this scenario, dubbed secluded DM, direct searches and direct DM production at accelerators are suppressed, and thus the stringent limits associated to these searches can be evaded. Instead, secluded DM can be tested with indirect detection methods: DM annihilates into dark mediators which in turn decay into SM particles. These processes can lead to unconventional indirect signatures. For instance, in standard WIMP scenarios, the gamma-rays injected by the annihilation of DM particles trapped inside the Sun are not detectable, since they are absorbed by the medium. Instead, in secluded DM models, long-lived mediators can escape, and decay outside the Sun. Ref. [31] presented a search for these signals with Fermi-LAT, assuming that the mediators decay into two photons. For mediators much lighter than DM, the resulting photon spectrum has a peculiar box-like shape. Therefore, the signal can be identified looking for features in the spectrum of gamma-rays from the Sun. The analysis is based on the ON/OFF technique (ON/OFF regions centered on the Sun and on the anti-Sun respectively) and exploits 10 years of data. Upper limits on signal fluxes have been derived. These results can be converted into bounds on a combination of the lifetime of the mediators, and on the DM-nucleon scattering cross section, which determines the amount of DM particles captured inside the Sun.

Bounds on secluded DM have been computed in [32] as well, for a variety of final states from the mediators decays. Future sensitivities for the CTA and SWGO experiments are presented.

As discussed in sec. 2.3 several proposed experiments plan to increase the sensitivity to gammaray fluxes at $\mathrm{MeV}$ energies. Refs.[17, 27] show that these proposals will be valuable probes for DM in the form of primordial black holes (PBHs). PBHs with masses in the range $\sim 10^{17}-10^{22} \mathrm{~g}$ can account for all the DM in the Universe. One way to test this scenario is to search for the fluxes of SM particles produced by the evaporation of PBHs. Limits from current data exclude the portion of parameter space in blue in fig. 3, testing PBHs masses up $\sim 10^{17} \mathrm{~g}$. For masses around the upper edge of the excluded region, the spectrum of primary photons from Hawking evaporation peaks at 

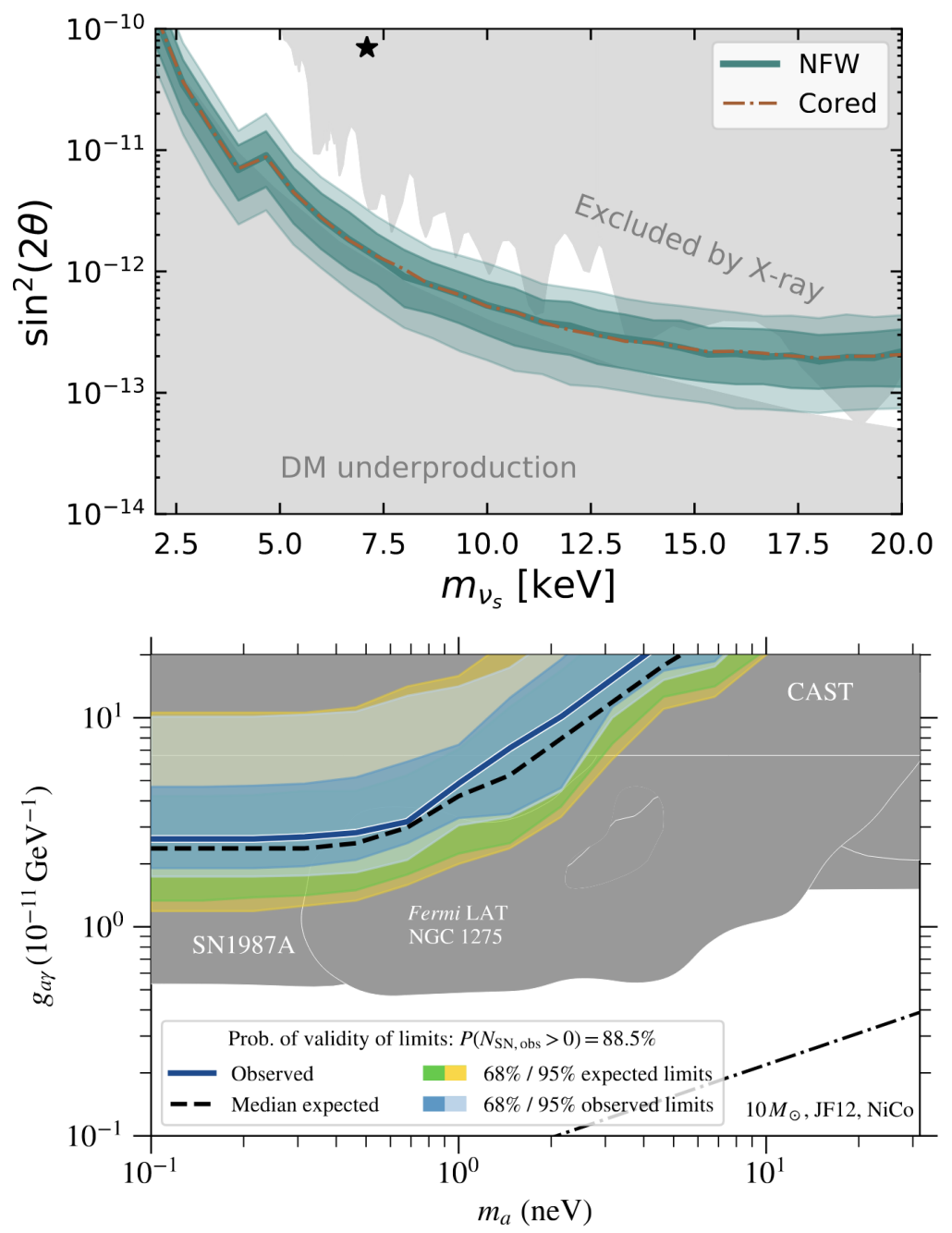

Figure 4: Top: Expected sensitivity of eROSITA on the mixing angle of sterile neutrinos with active neutrinos. Taken from [29]. Upper limits on the couplings of ALPs with photons from Fermi-LAT observations. Taken from [30].

energies in the $\mathrm{MeV}$ range. For this reason, future $\mathrm{MeV}$ gamma-ray experiments will be able to significantly improve current limits, covering new regions of the parameter space, see fig. 3.

Light PBHs with masses around $\sim 510^{14} \mathrm{~g}$ have a lifetime comparable to the age of the Universe. During the last stage of their evaporation process, these PBHs are predicted to emit short bursts of gamma-ray photons, which can be searched for with current gamma-ray instruments. Ref. [33] presented an analysis performed with H.E.S.S. data, looking for clusters of photons with timescales in the range of 10-120 seconds. Taking into account statistical fluctuations, no signals have been found. The upper limits on the PBH evaporation rate improve previous bounds, and complement the constraints from HAWC which test timescales of $\lesssim 10$ seconds.

Heavier PBHs, with masses in the range $\sim 10^{-1}-10^{2} M_{\odot}$, have been considered in [34]. In astrophysical environments these objects can accrete gas, which in turn produces non-thermal 
radiation at X-ray and radio frequencies. Ref. [34] considers a population of PBHs in the Central Molecular Zone, a region around the galactic center hosting a large reservoir of dense molecular clouds. Using observations of the NuSTAR X-ray telescope, and for certain assumptions on the accretion process, and on the PBHs density distribution, ref. [34] derives upper limits on the fraction of DM in forms of PBHs.

$\mathrm{X}$-ray telescopes are suitable instruments to test decaying DM candidates in the keV range, as sterile neutrinos and axion-like particles (ALPs) with $\sim \mathrm{keV}$ masses. These candidates are expected to produce monochromatic line signals through their decays. For instance, a sterile neutrino can decay into an active neutrino and a photon, a process occurring through the mixing with active neutrinos. Prospects for detection with eROSITA have been presented in [29] (see also [35] for the prospects of X-ray telescopes for DM signals from dwarf spheroidal galaxies). This experiment covers the energy range $0.3-10 \mathrm{keV}$, and has an excellent energy resolution, an important requirement to look for line signals. Exploiting the full sky coverage of eROSITA, [29] focuses on signals from the galactic halo, considering latitudes $|b|>20$ degrees. The sensitivity reach for sterile neutrinos is presented in fig. 4, for two choices of DM density profiles. The upper grey region is excluded by current X-ray data, while in the lower grey area sterile neutrinos are underabundant, assuming a specific production mechanism. As evident, this scenario is already quite constrained by observations, and eROSITA is expected to probe a significant part of the parameter space currently viable. The black star in fig. 4 highlights the best-fit value of the unidentified $3.5 \mathrm{keV}$ line. This emission was detected in X-ray observations, and it has been tentatively been interpreted in terms of sterile neutrino decays. The validity of this hypothesis in the light of current constraints (as well the nature of this line) is still under debate. eROSITA will be able to provide a robust test of the scenario. Furthermore, ref. [29] presented an analysis in terms of keV scale ALPs, in connection also with the excess of electron recoil events in the XENON1T data, see [29] for more details.

Ref. [30] considered very light DM candidates, namely ALPs with masses below $\sim 10 \mathrm{neV}$. These particles are predicted to interact with electromagnetic fields, in such a way that they can be produced in the interior of stars, like core-collapse supernova $(\mathrm{SN})$, through the conversion of photons. Most of the ALPs can freely escape from the star, and they can be reconverted back into photons through the magnetic field of our galaxy. Therefore, a signature of this scenario would be a burst of gamma-rays from core-collapse SNe, coincident in time with neutrinos, which, similarly to ALPs, are produced inside SNe and easily escape from the sources. Using neutrino and gamma-ray observations from the SN1987A, constraints on ALPs have been derived in the past. Ref. [30] exploits the current Fermi-LAT observations, and focuses on catalogues of extragalactic SNe. Given the distance of these sources, the associated neutrino fluxes have not been detected. Therefore, the explosion time is estimated in [30] using optical light curves. Under the assumption that at least one SN of the sample was in the Fermi-LAT field of view during its explosion (the probability was estimated to be around 89\%), ref. [30] derives constraints on the ALP coupling to photons, see fig. 4. These bounds are weaker than those from the SN1987A. However, promising prospects for improvement are expected with the extended sample of SNe that will be available in the near future. Let us mention that the cumulative emission of ALPs by the SNe in the Universe produces a diffuse ALPs background, which can be searched for with Fermi-LAT [36].

Finally, exotic DM candidates in form of nuclearites have been discussed in [37-43]. Nu- 
clearites are hypothetical macroscopic objects, composed by strange quark matter. These DM candidates travelling into the atmosphere are expected to loose part of their energy and produce light, appearing as meteors with unusual high speed. Differently from metors, nuclearites should show up mostly in the lower part of the atmosphere. Moreover, since their energy loss is expected to be small, their tracks have an almost constant brightness. Several experiments plan to search for nuclearites. Ref. [37] presented a search performed by the Pi of the Sky project, leading to constraints on the flux of nuclearites with masses from $10^{-4} \mathrm{~kg}$ up to $10^{2} \mathrm{~kg}$. These bounds improve previous limits in the literature for masses above $100 \mathrm{~g}$.

\section{DM searches with charged cosmic-rays}

\subsection{Cosmic-rays propagation}

Understanding the transport of charged cosmic-rays in the galactic environment is essential in order to look for exotic DM signals in the antimatter component of the cosmic-rays. In the past, the so-called MIN-MED-MAX propagation models have been proposed with the aim of bracketing the uncertainty on the DM fluxes arising from CR propagation. These benchmark models have been extensively used by the DM community since then. Ref. [45] presented an update in light of the wealth of new data appeared in the recent years. The analysis is based on a combined fit to measurements of the ratio of secondary to primary cosmic-ray species, and of unstable nuclei. The combination of all these data allows to constrain the parameters of the diffusion model, especially the size of the diffusive halo, which is a key ingredient for the prediction of DM signals. From a sample of models consistent with current data, [45] defines new MIN-MED-MAX models. These correspond to the minimal, median, and maximal fluxes of antimatter produced by DM annihilations. For example, fig. 5 shows the antiproton DM signals predicted by these benchmark models. With respect to the original MIN-MED-MAX models, the uncertainties on DM signals are reduced by a factor 3-4 (5) for positrons (antiprotons).

\subsection{Positrons}

A rise in the positron fraction above $10 \mathrm{GeV}$ has been observed by the AMS-02 (and previously by PAMELA) experiment. Although its origin is still under debate, recent observations support an explanation based on a primary positron component produced by pulsars. Under this assumption, [44] derived strong constraints on DM annihilations into leptonic channels. The main idea is that the positron signal produced by these leptonic final states has an hard spectrum, which, on the top of a smooth positron flux produced by pulsars, leads to spectral features in the positron fraction. In more details, ref. [44] considers a model based on the DM signal and an astrophysical background component, which includes the contribution from pulsars. The model is fitted to the positron, proton and Helium data from AMS-02. This procedure allows to constrain the model parameters, and obtain consistent upper limits on the DM signal. [44] improves over a previous similar analysis using more recent data, implementing a more sophisticated model for solar modulation, and exploring in more details the uncertainty related to the cosmic-ray propagation. The limits on the DM annihilation cross-section, presented in fig. 5, exclude thermal relic cross-sections for masses up to $60-380 \mathrm{GeV}$, depending on the channel. 

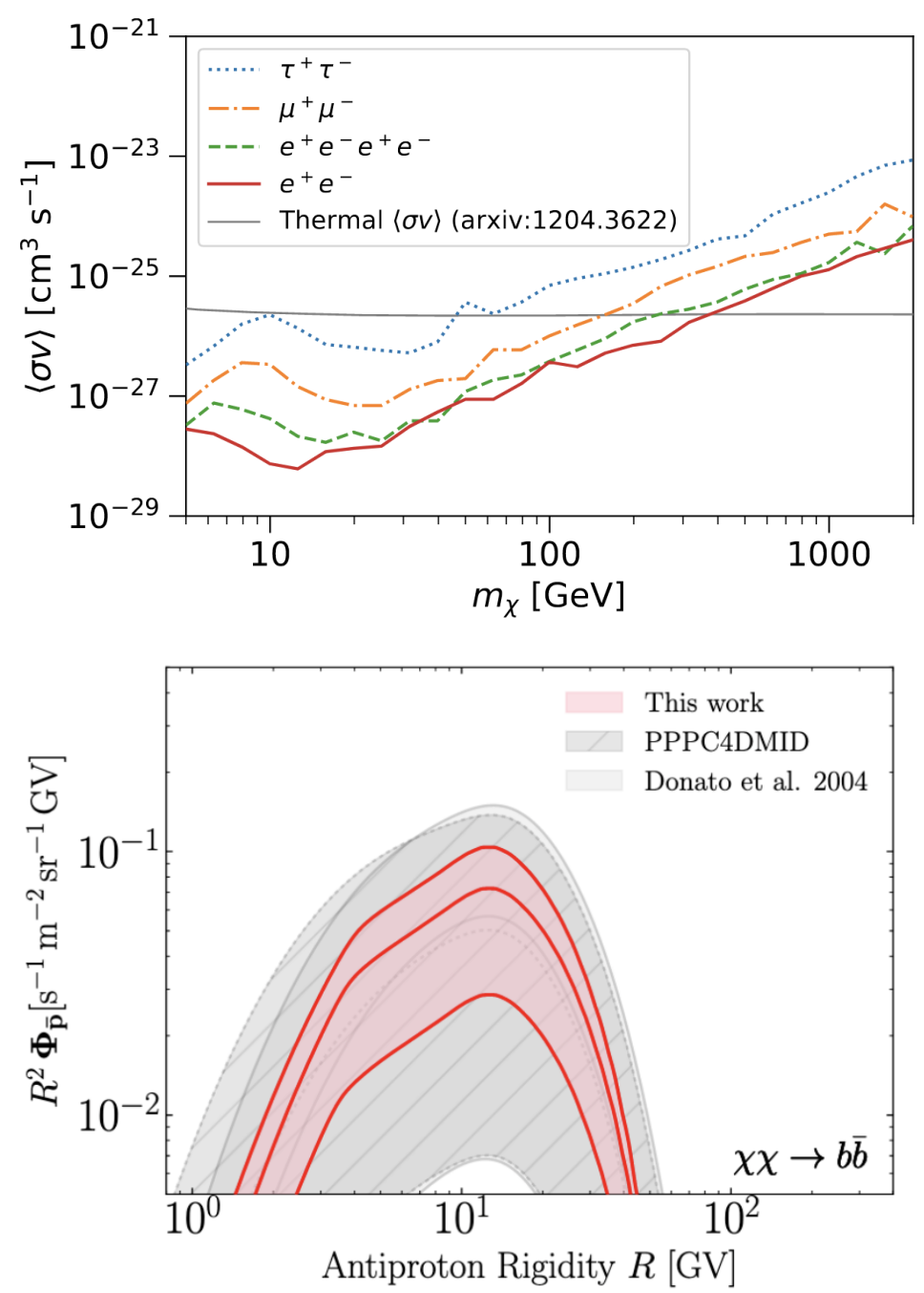

Figure 5: Top: Upper limits on the DM annihilation cross-section into different leptonic channels from a search of positron signals in the cosmic-ray data. Taken from [44]. Bottom: The red lines show the flux of anti-protons from DM annihilations predicted by the MIN-MED-MAX propagation models in [45]. The gray area refers to the predictions of the original MIN-MED-MAX models. Taken from [45].

\subsection{Antiprotons}

At ICRC 2021, a discussion on the AMS-02 antiproton excess has been presented in [46, 47]. This anomaly corresponds to an excess of antiprotons at rigidities around $10 \mathrm{GV}$, which can potentially be explained by DM annihilations. Interestingly, the required values of DM masses and annihilation cross-sections are in the ballpark of those favoured by the galactic center $\mathrm{GeV}$ excess discussed in sec.2.2. Several systematic uncertainties are relevant to assess the statistical significance of the excess, in particular those related to the cosmic-ray propagation, the uncertainty on the antiproton production cross-section, and on the AMS-02 measurements. Concerning the latter ingredient, the correlation structure of the AMS-02 systematic errors is not publicly available yet. This has motivated the analysis of [46], which reported an evaluation of the covariance matrix 
of the AMS-02 systematic errors. A particular emphasis has been devoted to the uncertainty on the cross section for cosmic-ray absorption in the detector, which is the main systematics in the AMS-02 data in the relevant rigidity range. This component has been modelled exploiting nuclear scattering measurements, and using a theoretical nuclear model to fit these data. Using these results, [46] has then proceeded to reevaluate the significance of the antiproton excess, finding that it reduces below $1 \sigma$, once all the systematics are properly taken into account. The broad conclusions are in agreement with the results of other analysis in the literature.

Clearly, it is very important to reduce systematic uncertainties to search for faint DM signals in cosmic-ray data. In this respect, experimental efforts to measure cosmic-ray cross sections at accelerators are precious, see e.g [48].

\subsection{Antideuterons and antihelium}

The General Antiparticle Spectrometer (GAPS) is an experiment designed to detect low energy ( $\lesssim 0.25 \mathrm{GeV} / \mathrm{n}$ ) cosmic-ray antiprotons and antinuclei, see [49, 51, 52]. The antideuteron channel is particularly exciting for DM searches since it is almost background free at low energies. As an example, fig. 6 shows the antideuteron flux from DM annihilations, and from the interaction of cosmic-rays with the interstellar medium (including the secondary and tertiary components). As evident, the signal and the backgrounds peak at different energies, because of the different kinematics involved in the relevant processes. Moreover, at low energy, the background can be extremely suppressed with respect to a potential DM signal. Fig. 5 reported also the future sensitivity for GAPS: DM models like those motivated by the antiproton excess can be tested with future antideuteron searches. The first flight of GAPS is expected in late 2022.

Searches of DM signals with antihelium are usually expected to be less promising. However, ref. [50] pointed out a production mechanism which can potentially boost the DM signal of orders of magnitude. This corresponds to the decays of the $\bar{\Lambda}_{b}$ baryon, a channel overlooked in previous analysis. The idea is that $\bar{\Lambda}_{b}$ baryons can be sizeably produced from the hadronization of $\bar{b}$ quarks produced by DM annihilations. Given its quantum numbers and its mass, the $\bar{\Lambda}_{b}$ baryon is prone to decay into low energies antinucleons, which can merge into antihelium. As shown in fig. 6, this channel can largely increase the antihelium signal, leading to a flux potentially detectable by future AMS-02 measurements. Unfortunately, there are large uncertainties in the predictions, which call for dedicated experimental campaigns in order to measure the branching ratio $\operatorname{Br}\left(\bar{\Lambda}_{b} \rightarrow \overline{\mathrm{He}}\right)$.

\section{DM searches with neutrinos}

\subsection{Neutrinos from the galactic halo}

Ref. [53] presented a search for DM with neutrinos from the galactic center region with ANTARES. ANTARES is an underwater neutrino detector in the mediterranean sea consisting of 12 detector lines at a depth of $2.5 \mathrm{~km}$. The analysis in [53] exploits data between 2007 and 2020 . It is based on a search for neutrino events clustered around the galactic center region, as expected for DM annihilations, on the top of a background of atmospheric neutrino events. No excesses have been found, and upper limits on the DM annihilation cross-section have been derived, see fig. 7. Future sensitivities for the KM3NeT experiment are presented as well in [53]. KM3NeT is 

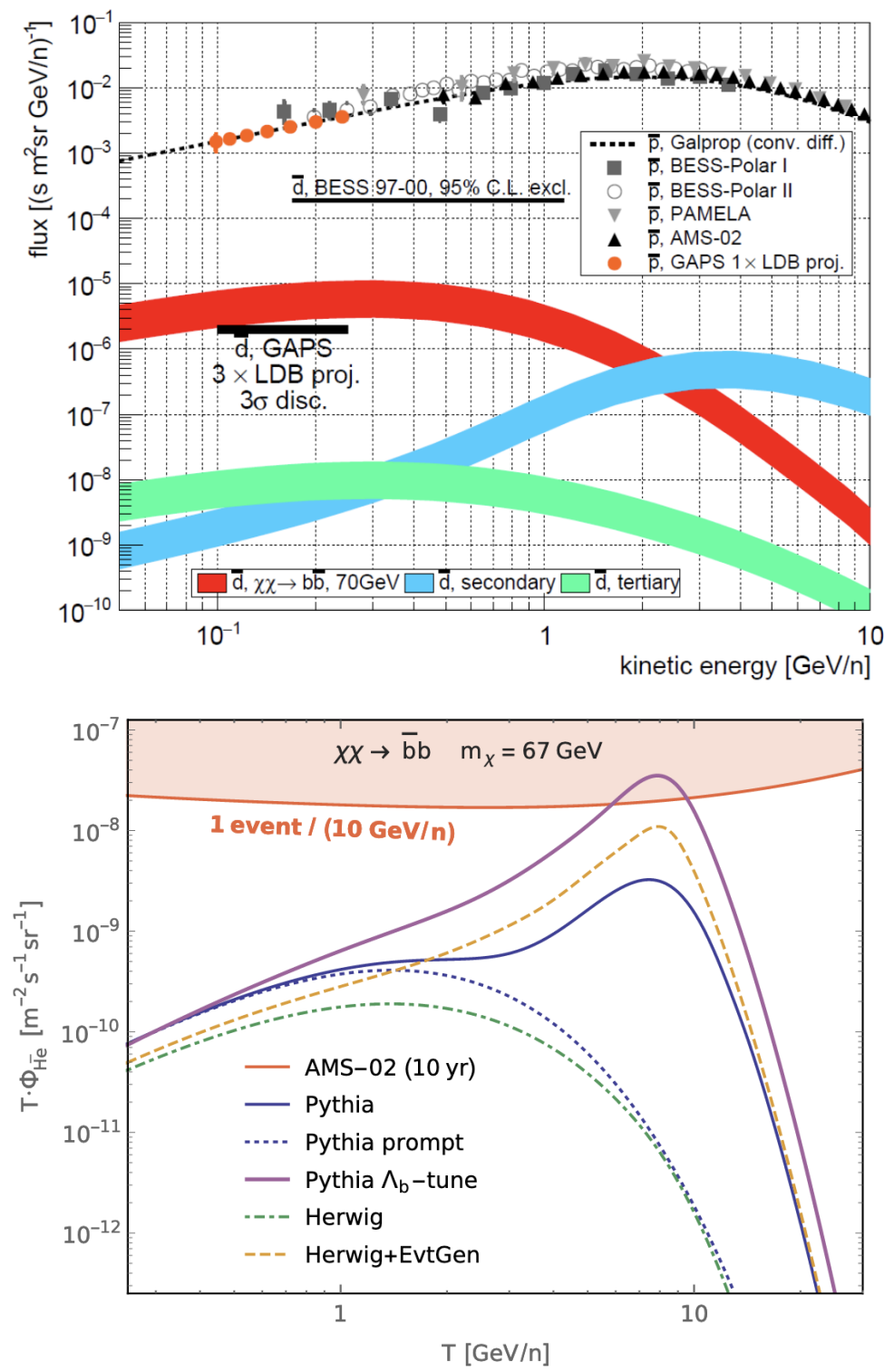

Figure 6: Top: Antideuteron flux from DM (red) and from the astrophysical background (green and blue). The projected sensitivity of the GAPS experiment is shown in black. Taken from [49]. Bottom: Flux of antihelium from DM annihilations and the AMS-02 antihelium sensitivity. Taken from [50].

currently under construction, and it is is based on an array of light sensors located in two sites in the mediterranean sea. As shown in fig. 7, it will significantly improve current results of ANTARES.

An analogous search for IceCube has been discussed in [55]. IceCube is a neutrino detector in the antarctic ice at the South Pole. Sensitivities on the DM annihilation cross-section, and for DM with masses up to $1 \mathrm{TeV}$ have been presented. Other DM searches with IceCube have been reported in $[56,57]$, focussing respectively on the GC region and on clusters of galaxies. 

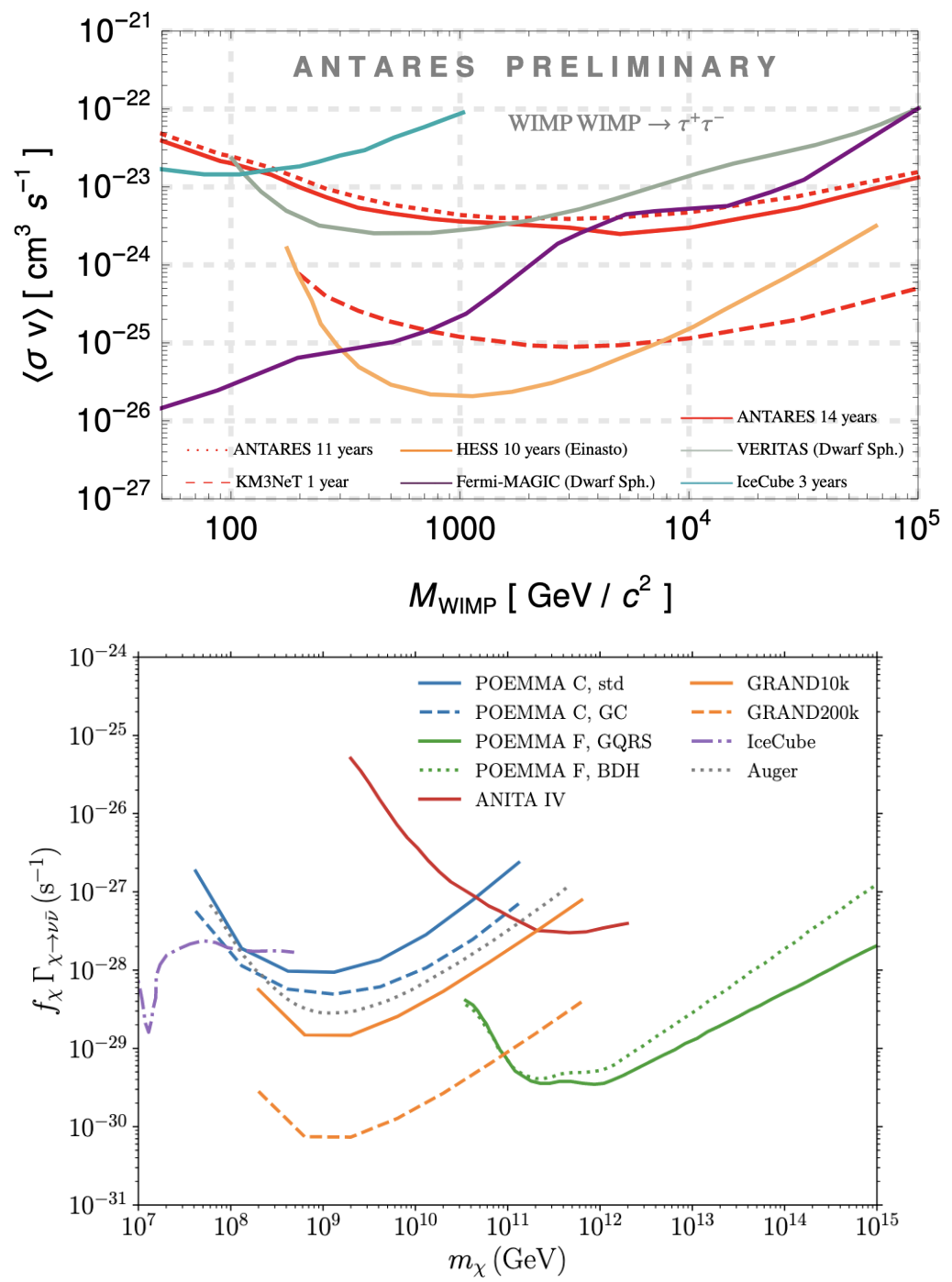

Figure 7: Top: Constraints on the DM annihilation cross-section from a search performed with ANTARES. Upper limits from other experiments, and previous ANTARES searches are also shown. The red-dashed line shows the expected sensitivity of KM3NeT. Taken from [53]. Bottom: Expected sensitivity of POEMMA and GRAND to the DM decay width into neutrinos. Bounds from other experiments are shown as well. Taken from [54].

\subsection{Neutrinos from the Sun and the Earth}

Dark matter travelling through the Sun and the Earth can scatter with the material and loose enough energy to be gravitationally captured. Therefore, a population of DM particles accumulates in the interior of these objects. DM annihilations can produce neutrinos, which can escape from these systems, and they can finally be detected by neutrino experiments. If the annihilation rate and the capture rate are sizeable enough, an equilibrium is attained in a short timescale (meaning that it is shorter than the age of the object). Under this condition, the DM neutrino signal is insensitive to the DM annihilation cross-section, and it depends instead on the DM scattering cross-section with 
nuclei (which controls the capture rate). The latter is the same quantity probed by direct detection searches, although obviously with a very different technique. An overview of DM searches from the Sun with IceCube has been presented in [58]. For Spin Dependent (SD) interactions, IceCube limits are competitive with those from direct detection experiments. For Spin Independent (SI) interactions, direct detection constraints are stronger. This is due to the coherent enhancement of the SI interactions, which favours targets with heavy nuclei (the Sun is mainly composed by light nuclei). SI and SD interactions are not the only possible DM interactions with nuclei. A nonrelativistic effective field theory has been put forward in order to cover all the possibilities. Ref. [59] presented an analysis where DM searches from the Sun with IceCube have been reinterpreted in this framework. Upper limits on the operators of the effective field theory are presented, and compared with those from direct detection. The two methods are highly complementary, and one is more sensitive than the other for certain operators and DM masses.

DM searches with neutrinos have been discussed also in [24, 60, 61, 63, 64]. [60] reported on a search for dark matter from the Earth with IceCube. The sensitivities on SI interactions from this analysis improve previous IceCube results, although limits from direct detection are stronger.

\subsection{Ultra-high energy neutrinos}

Several experiments have been proposed in order to detect ultra-high energy neutrinos, such as POEMMA, GRAND, RNO-G and the radio array of IceCube-Gen2. POEMMA is designed to detect the Cherenkov signals from cosmic neutrinos with energies above $\sim 10^{7} \mathrm{GeV}$. GRAND plans to exploit the radio signals associated to air showers, using an array of radio antennas. Other two proposed radio detectors are RNO-G, and a radio array to be installed in IceCube-Gen2. Sensitivities of these experiments for DM searches with neutrinos have been presented in [54, 62]. Depending on the decay channel, these experiments have the potential to increase the sensitivity of current experiments to heavy decaying DM candidates, see fig. 7.

\section{Direct detection searches}

Updates on the status of several direct detection experiments have been presented at ICRC 2021. DEAP-3600 is a single phase liquid argon detector, employing $3300 \mathrm{~kg}$ of target material [66]. The current sensitivity of DEAP-3600 to standard SI interactions is weaker than those reached by some other experiments, unless for specific scenarios of isospin violation. Upgrades to reduce backgrounds are in progress. An update on the status of the DARWIN project has been reported in [65]. DARWIN is a proposed double phase Xenon detector consisting of 40 tonnes of active material. This experiment will improve current sensitivities reaching the so-called neutrino floor, i.e. the level of sensitivities at which astrophysical neutrinos will act as an irreducible backgrounds for this experiment. DARWIN will allow also to study of other interesting signals, such as neutrinoless double beta decays, solar neutrinos, axions and dark photons. Noble liquid experiments with large exposures and low backgrounds, like DARWIN, have (or will have) leading sensitivities for WIMPs with masses above $\gtrsim 5-10 \mathrm{GeV}$. Lighter masses are particularly difficult to explore, given the small amount of energy deposited by DM in the collisions. DAMIC is an experiment designed to search for light DM ( $\lesssim 10 \mathrm{GeV}$ ). It is located at SNOLAB and it consists of CCDs. Ref. [69] presented an analysis based on a $11 \mathrm{~kg} \cdot \mathrm{d}$ exposure dataset. The distribution of events is compatible 
with backgrounds for energies above $200 \mathrm{eV}_{\text {ee }}$, while an excess is present at lower energies. Its origin is still under investigation. The upper limits on the WIMP-nucleon cross section derived in this analysis are complementary to those from other experiments. The successor of DAMIC will be DAMIC-M, which will benefit from a 25 times larger detector mass, and from a better CCD technology, which will allow an improved readout noise [67]. The relevance of novel CCDs for direct detection is discussed in [68].

\section{Conclusions}

In conclusion, at ICRC 2021 clear non-gravitational signals of DM have not emerged. On the other hand, prospects for detection with forthcoming experiments are encouraging. Furthermore, anomalies in astrophysical data, and in direct searches are under close examination. Overall, the field is experiencing a steady progress, which is hopefully bringing us closer to a future detection.

\section{Acknowledgments}

I would like to thank the organizers and all the participants for the stimulating conference. I am very grateful to the organizers for the invitation to present the dark matter rapporteur talk. I acknowledge support from the INFN grant "LINDARK," the research grant "The Dark Universe: A Synergic Multimessenger Approach No. 2017X7X85" funded by MIUR, and the project "Theoretical Astroparticle Physics (TAsP)" funded by the INFN.

\section{References}

[1] H. Abdalla, F. Aharonian, F. Ait-Benkhali, O. Anguener, C. Arcaro, C. Armand et al., Combined dark matter searches towards dwarf spheroidal galaxies with Fermi-LAT, HAWC, H.E.S.S., MAGIC, and VERITAS, PoS ICRC2021 (2021) 528.

[2] C. Maggio, D. Kerszberg, D. Ninci, V. Vitale, V. A. Acciari, S. Ansoldi et al., Upper limits on the WIMP annihilation cross section from a joint analysis of dwarf spheroidal satellite galaxy observations with the MAGIC telescopes, PoS ICRC2021 (2021) 512.

[3] C. Giuri, VERITAS dark matter search in dwarf Spheroidal galaxies: an extended source analysis, PoS ICRC2021 (2021) 515.

[4] J. Coronado-Blázquez, M. Doro, M. A. Sánchez-Conde and A. Aguirre-Santaella, Sensitivity of the Cherenkov Telescope Array to dark subhalos, PoS ICRC2021 (2021) 544.

[5] J. Coronado-Blázquez, M. A. Sánchez-Conde, J. Pérez-Romero and A. Aguirre-Santaella, Spatial extension as a "smoking gun" for dark subhalo detection with Fermi-LAT and implications for WIMP constraints, PoS ICRC2021 (2021) 508.

[6] V. Gammaldi, J. Coronado-Blázquez, M. A. Sánchez-Conde and B. Zaldivar, Searching for dark matter in Fermi-LAT unidentified sources with Neural Network, PoS ICRC2021 (2021) 493. 
[7] M. Stref, T. Lacroix, G. Facchinetti, J. Pérez-Romero, J. LAVALLE, D. Maurin et al., Classification of targets for gamma-ray dark matter searches with velocity-dependent annihilation and substructure boost, PoS ICRC2021 (2021) 571.

[8] A. Montanari, E. Moulin, D. Glawion, H. Abdalla, F. Aharonian, F. Ait-Benkhali et al., Search for dark matter annihilation signals from unidentified Fermi-LAT objects with H.E.S.S., PoS ICRC2021 (2021) 538.

[9] A. Aguirre-Santaella, M. Sánchez-Conde, R. Angulo, G. Ogiya and J. Stücker, Shedding light on low-mass subhalo survival with numerical simulations, PoS ICRC2021 (2021) 561.

[10] V. Gammaldi, J. Pérez-Romero, J. Coronado-Blázquez, M. Di Mauro, M. A. Sánchez-Conde, O. b. o. the Fermi Large Area Telescope Collaboration et al., Dark Matter search in dwarf irregular galaxies with the Fermi Large Area Telescope, PoS ICRC2021 (2021) 509.

[11] H. Abdalla, F. Aharonian, F. Ait-Benkhali, O. Anguener, C. Arcaro, C. Armand et al., Search for dark matter annihilation towards the inner Milky Way halo with the H.E.S.S. Inner Galaxy Survey, PoS ICRC2021 (2021) 511.

[12] A. Babic, A. Baquero, I. Batković, J. Becerra Gonzalez, J. Besenrieder, H. Bökenkamp et al., Search for Gamma-ray Line emission from Dark Matter annihilation in the Galactic Centre with the MAGIC telescopes, PoS ICRC2021 (2021) 520.

[13] M. Di Mauro, Multimessenger constraints on the dark matter interpretation of the Fermi-LAT Galactic center excess, ICRC (2021) .

[14] J. Berteaud, F. Calore and M. Clavel, A multi-wavelength search for bulge millisecond pulsars, PoS ICRC2021 (2021) 681.

[15] O. Macias, H. van Leijen, D. Song, S. Ando, S. Horiuchi and R. M. Crocker, CTA sensitivity to the high-energy tail of the Fermi GeV excess, PoS ICRC2021 (2021) 562.

[16] A. Viana, A. Albert, J. P. Harding, J. Hinton, H. Schoorlemmer and V. de Souza, Searching for Dark Matter with the Southern Wide-field Gamma-ray Observatory (SWGO), PoS ICRC2021 (2021) 555.

[17] J. LeyVa, Exploring MeV gamma rays from dark matter annihilation and evaporating primordial black holes in the GRAMS experiment, PoS ICRC2021 (2021) 552.

[18] T. Slatyer, Dark Matter: Knowns and Unknowns, ICRC (2021) .

[19] P. Harding, An Optimized Search for Dark Matter in the Galactic Halo with HAWC, ICRC (2021) .

[20] A. U. Abeysekara, A. Albert, R. Alfaro, C. Alvarez, J. d. D. Álvarez Romero, J. R. Angeles Camacho et al., Limits on Diffuse Dark Matter with HAWC, PoS ICRC2021 (2021) 529. 
[21] M. Nisa, A. U. Abeysekara, A. Albert, R. Alfaro, C. Alvarez, J. d. D. Álvarez Romero et al., Search for TeV decaying dark matter from the Virgo cluster of galaxies, PoS ICRC2021 (2021) 530 .

[22] M. Chianese, Constraints on decaying dark matter with LHAASO-KM2A, ICRC (2021) .

[23] A. Addazi, K. Belotsky, V. Beylin, M. Cirelli, G. DiSciascio, A. Esmaili et al., Dark Matter Searches for heavy Dark Matter with LHAASO, PoS ICRC2021 (2021) 574.

[24] B. Skrzypek, C. Arguelles and M. Chianese, Decaying Dark Matter at IceCube and its Signature in High-Energy Gamma-Ray Experiments, PoS ICRC2021 (2021) 566.

[25] C. Eckner, Sensitivity of the Cherenkov Telescope Array to a dark matter signal from the Galactic centre, PoS ICRC2021 (2021) 547.

[26] J. Perez-Romero, Sensitivity of CTA to gamma-ray emission from the Perseus galaxy cluster, PoS ICRC2021 (2021) 546.

[27] S. Profumo, Hunting for Dark Matter and New Physics with GECCO, ICRC (2021) .

[28] E. Pinetti, M. Cirelli, B. Kavanagh and N. Fornengo, Integral X-ray constraints on sub-GeV dark matter, PoS ICRC2021 (2021) 563.

[29] A. Dekker, E. Peerbooms, F. Zimmer, K. C. Y. Ng and S. Ando, Probing sterile neutrinos and axion-like particles from the Galactic halo with eROSITA, PoS ICRC2021 (2021) 556.

[30] M. Meyer and T. Petrushevska, Extending the sample of core-collapse supernovae for searches of axion-like-particle induced gamma-ray bursts with the Fermi LAT, PoS ICRC2021 (2021) 510.

[31] F. Loparco, D. Serini and M. N. Mazziotta, Indirect Dark Matter searches in the gamma-ray channel toward the Sun with the Fermi LAT, PoS ICRC2021 (2021) 513.

[32] C. Siqueira, A. Viana, F. queiroz and G. Fortes, Indirect Searches for Secluded Dark Matter, PoS ICRC2021 (2021) 577.

[33] T. Tavernier, J.-F. Glicenstein, F. Brun, V. Marandon, H. Abdalla, F. Aharonian et al., Limits on primordial black hole evaporation from H.E.S.S. observations., PoS ICRC2021 (2021) 518.

[34] F. Scarcella, D. Gaggero and J. Garcia-Bellido, Searching for isolated black holes in the Milky Way, PoS ICRC2021 (2021) 565.

[35] F. Zimmer, E. Peerbooms and S. Ando, Decaying dark matter in dwarf spheroidal galaxies: Prospects for X-ray and gamma-ray telescopes, PoS ICRC2021 (2021) 567.

[36] C. Eckner, F. Calore, P. Carenza, M. Giannotti, J. Jaeckel, F. Sivo et al., Constraining the diffuse supernova axion-like-particle background with high-latitude Fermi-LAT data, PoS ICRC2021 (2021) 543. 
[37] L. Piotrowski, K. Małek, L. Mankiewicz, M. Sokołowski, G. Wrochna, A. Zadrożny et al., Limits on the flux of heavy compact objects from the the "Pi of the Sky" project, PoS ICRC2021 (2021) 536.

[38] T. C. Paul, S. T. Reese, L. A. Anchordoqui and A. V. Olinto, EUSO-SPB2 sensitivity to macroscopic dark matter, PoS ICRC2021 (2021) 519.

[39] S. Abe, M. Arahori, D. Barghini, M. E. Bertaina, M. Casolino, A. Cellino et al., DIMS Experiment for Dark Matter and Interstellar Meteoroid Study, PoS ICRC2021 (2021) 554.

[40] D. Barghini, S. Valenti, S. Abe, M. Arahori, M. E. Bertaina, M. Casolino et al., Characterization of the DIMS system based on astronomical meteor techniques for macroscopic dark matter search, PoS ICRC2021 (2021) 500.

[41] M. Bouta, A. Moussa, Y. Tayalati, J. Brunner and G. Emilia Pavalas, Search for nuclearites with the ANTARES detector, PoS ICRC2021 (2021) 532.

[42] D. Shinto, Y. Iwami, M. Fujioka, Y. Tameda, K. Nadamoto, F. Kajino et al., Solar Power Supply and Environmental Control System for DIMS Experiment, PoS ICRC2021 (2021) 502.

[43] L. Piotrowski, D. Barghini, M. Battisti, A. S. Belov, M. E. Bertaina, F. Bisconti et al., Towards observations of nuclearites in Mini-EUSO, PoS ICRC2021 (2021) 503.

[44] I. John and T. Linden, Cosmic-Ray Positrons Strongly Constrain Leptophilic Dark Matter, PoS ICRC2021 (2021) 568.

[45] P. Salati, M. Boudaud, M. Cirelli, L. Derome, Y. Génolini, J. LAVALLE et al., New cosmic ray MIN-MED-MAX benchmark models for dark matter indirect signatures, PoS ICRC2021 (2021) 572.

[46] J. Heisig, M. Korsmeier and M. W. Winkler, Revisiting the AMS-02 antiproton excess: The role of correlated errors, PoS ICRC2021 (2021) 575.

[47] P. De la Torre Luque, D. Gaggero and M. N. Mazziotta, Antiproton production from cosmic-ray interactions and its compatibility with AMS-02 data, PoS ICRC2021 (2021) 570.

[48] M. Naskret, Light (anti)nuclei production cross section studies in $p+C$ collisions at the NA61/SHINE experiment., PoS ICRC2021 (2021) 535.

[49] M. Xiao, In Search of Cosmic-Ray Antinuclei from Dark Matter with the GAPS Experiment, PoS ICRC2021 (2021) 494.

[50] M. Winkler, A detectable antihelium flux from dark matter annihilation, PoS ICRC2021 (2021) 578 .

[51] A. Stoessl, Searching for cosmic antihelium nuclei with the GAPS experiment, PoS ICRC2021 (2021) 499. 
[52] A. Tiberio, T. Aramaki, R. Bird, M. Boezio, S. E. Boggs, V. Bonvicini et al., Reconstruction of antinucleus-annihilation events in the GAPS experiment, PoS ICRC2021 (2021) 504.

[53] M. Ageron, S. Aiello, A. Albert, M. Alshamsi, S. Alves Garre, Z. Aly et al., Indirect dark matter searches with neutrinos from the Galactic Centre region with the ANTARES and KM3NeT telescopes, PoS ICRC2021 (2021) 537.

[54] C. Guepin, R. Aloisio, L. A. Anchordoqui, A. Cummings, J. Krizmanic, A. V. Olinto et al., Probing the properties of superheavy dark matter annihilating or decaying into neutrinos with ultra-high energy neutrino experiments, PoS ICRC2021 (2021) 551.

[55] R. Abbasi, M. Ackermann, J. Adams, J. Aguilar, M. Ahlers, M. Ahrens et al., Indirect search for dark matter in the Galactic Centre with IceCube, PoS ICRC2021 (2021) 524.

[56] A. McMullen, C. Arguelles, A. Schneider, A. Vincent, R. Abbasi, M. Ackermann et al., Searching for Dark Matter Neutrino Scattering in the Galactic Centre with IceCube, PoS ICRC2021 (2021) 569.

[57] R. Abbasi, M. Ackermann, J. Adams, J. Aguilar, M. Ahlers, M. Ahrens et al., A Search for Neutrinos from Decaying Dark Matter in Galaxy Clusters and Galaxies with IceCube, PoS ICRC2021 (2021) 506.

[58] J. Lazar, Searching for Dark Matter from the Sun with Ten Years of IceCube, ICRC (2021).

[59] R. Abbasi, M. Ackermann, J. Adams, J. Aguilar, M. Ahlers, M. Ahrens et al., Constraining non-standard Dark Matter-Nucleon Interactions with IceCube, PoS ICRC2021 (2021) 522.

[60] R. Abbasi, M. Ackermann, J. Adams, J. Aguilar, M. Ahlers, M. Ahrens et al., Search for dark matter from the center of the Earth with 8 years of IceCube data, PoS ICRC2021 (2021) 526.

[61] C. Toennis, R. Abbasi, M. Ackermann, J. Adams, J. Aguilar, M. Ahlers et al., Search for secluded dark matter with 6 years of IceCube data - Christoph Toennis, PoS ICRC2021 (2021) 521.

[62] R. Hajjar, Testing the stability of heavy dark matter with up-coming radio neutrino telescopes, PoS ICRC2021 (2021) 573.

[63] A. Cheek, S. Basegmez du Pree, C. Arina, M. Chianese, A. Dekker and S. Ando, Dark Matter Phenomenology from Upcoming Neutrino Telescopes:, PoS ICRC2021 (2021) 550.

[64] A. Kheirandish, C. A. Argüelles, A. Diaz, I. Safa and A. C. Vincent, Dark Matter Annihilation to Neutrinos: Current Limits and Future Prospects, PoS ICRC2021 (2021) 542.

[65] K. Thieme, DARWIN - a next-generation liquid xenon observatory for dark matter and neutrino physics, PoS ICRC2021 (2021) 548.

[66] M. Stringer, The DEAP-3600 Experiment, PoS ICRC2021 (2021) 527. 
[67] C. De Dominicis and M. Settimo, Simulations and background estimates for the DAMIC-M experiment, PoS ICRC2021 (2021) 553.

[68] A. M. Botti, M. Cababie, J. Estrada, G. Fernandez-Moroni, M. Sofo-Haro and J. Tiffenberg, Sub-GeV dark matter and neutrino searches with Skipper-CCDs: status and prospects., PoS ICRC2021 (2021) 505.

[69] M. Traina, A. Aguilar-Arevalo, D. Amidei, I. Arnquist, D. Baxter, G. Cancelo et al., Results on low-mass weakly interacting massive particles from a $11 \mathrm{~kg}$ d target exposure of DAMIC at SNOLAB, PoS ICRC2021 (2021) 539. 